BULLETIN OF THE

AMERICAN MATHEMATICAL SOCIETY

Volume 78, Number 5, September 1972

\title{
ON THE 2-SPHERES IN A 3-MANIFOLD
}

\author{
BY F. LAUDENBACH
}

Communicated by Raoul Bott, February 10, 1972

We shall give here an abstract of [2]. In this paper, the manifolds and the maps are $C^{\infty}$. Let us recall some definitions:

(1) Let $S$ and $S^{\prime}$ be two spheres in a 3-manifold $V^{3} ; S$ and $S^{\prime}$ are said to be homotopic if there exist two embeddings $\varphi, \varphi^{\prime}: S^{2} \rightarrow V$ with $S$ and $S^{\prime}$ as images that are homotopic in the family of smooth maps $S^{2} \rightarrow V . S$ and $S^{\prime}$ are said to be isotopic if there exists a diffeomorphism $H$ of $V$, isotopic to the identity, such that $H(S)=S^{\prime}$.

(2) $h: S^{2} \times[0,1] \rightarrow V$ is said to be a homotopy of disjunction of $S^{\prime}$ from $S$ if $h \mid S^{2} \times\{0\}$ is an embedding with $S^{\prime}$ as image and if $h\left(S^{2} \times\{1\}\right) \subset V-S$.

(3) $V$ satisfies the Poincare conjecture if any compact contractible 3-manifold of $V$ is diffeomorphic to $D^{3}$.

We obtain the following results:

THEOREM I. Let $V$ be a 3-manifold satisfying the Poincare conjecture and let $S, S^{\prime}$ be two spheres in $V$. If there exists a homotopy of disjunction of $S^{\prime}$ from $S$, then there exists an isotopy of disjunction.

THEOREM II. With the same hypotheses as in Theorem I, if $S$ and $S^{\prime}$ are homotopic, then $S$ and $S^{\prime}$ are isotopic.

THEOREM III. For any positive integer $p$, we shall denote $p \# S^{1} \times S^{2}$ the connected sum of $p$ copies of $S^{1} \times S^{2}$. Let $H$ be a diffeomorphism of $p \# S^{1} \times S^{2}$ homotopic to the identity. Then $H$ is isotopic to the identity.

REMARK. Theorems I and II are trivial if $S$ is null homotopic, because then $S$ is the boundary of a ball; hence we suppose that $S$ is not null homotopic.

THEOREM I $\Rightarrow$ THEOREM II. If $S^{\prime}$ is homotopic to $S$, then there exists a disjunction homotopy of $S^{\prime}$ from $S$. After Theorem I, there exists an isotopy of disjunction. If now $S$ and $S^{\prime}$ are homotopic and disjoint, they bound an $h$-cobordism, which is trivial, since $V$ satisfies the Poincare conjecture.

THEOREM II $\Rightarrow$ THEOREM III. Let $\Sigma_{1}, \ldots, \Sigma_{p}$ be the $p$ transversal spheres in the index 1 handlebodies of $p \# S^{1} \times S^{2}$. We can, by using mainly Theorem II, reduce to the case where $H \mid \Sigma_{1} \cup \ldots \cup \Sigma_{p}$ is the

AMS 1970 subject classifications. Primary 55A99, 57A35, 58D05.

Key words and phrases. Homotopy, isotopy, diffeomorphism of 3-manifold.

Copyright (C) American Mathematical Society 1972 
identity. To conclude, the main tools are the fibration theorems of Cerf and $\pi_{0}\left(\operatorname{Diff}^{+}\left(D^{3}\right)\right)=0 \quad[1]$.

Demonstration plan for Theorem I. In a first part, we study the following problem: let $f: S^{2} \rightarrow V$ be a map transversal to a sphere $S$ embedded in $V$; what are the obstructions to finding a homotopy of $f$ to a map $f^{\prime}: S^{2} \rightarrow V-S^{2}$ ?

In a second part, we introduce a technique of cuttings and glueings of spheres; it is a generalisation of the fact that the embedded connected sum of two spheres depends, to an isotopy, only on the homotopy class of the glueing path.

In these two parts, Poincare's hypothesis, is not used; we will indicate later on how it interferes in Theorem I's own proof.

Obstruction theory. Being given $f: S^{2} \rightarrow V$ transversal to $S$, we may consider the stratification of $S^{2}$ defined by $f^{-1}(S)$; we shall denote by $\Gamma(f)$ its nerve; $\Gamma(f)$ is a tree. If a transversal orientation of $S$ in $V$ is fixed, we obtain a natural orientation of the edges of $\Gamma(f)$. We shall complete the structure of $\Gamma(f)$ with weights assigned to the edges and to the pairs of edges.

The following notation will be used: for any component $C$ of $f^{-1}(S)$, $A(C)$ denotes the corresponding edge on $\Gamma(f)$. The transversally oriented curve $C$ bounds in $S^{2}$ a disk $D_{+}$, in which the positive normal enters, and a disk $D_{-}$, out of which it goes; $D_{+}$and $D_{-}$are oriented as $S^{2}$. We shall name $\alpha_{+}(A(C))$ and $\alpha_{-}(A(C))$ the homotopy classes of $f \mid D_{+}$ and $f \mid D_{-}$among the mappings $\left(D^{2}, \partial D^{2}\right) \rightarrow(V, S)$; the set of the homotopy classes of these mappings will be noted $\tilde{\pi}_{2}(V, S)$.

As $S$ is 1-connected, there exists on $\tilde{\pi}_{2}(V, S)$ a natural structure of an abelian group.

On the other hand, if $C$ and $C^{\prime}$ are two components of $f^{-1}(S)$, we choose an oriented path $\gamma$ joining $C$ to $C^{\prime}$ in $S^{2}$. We denote by $\omega\left(A(C), A\left(C^{\prime}\right)\right)$ the homotopy class of $f \mid \gamma$ among the mappings $([0,1]$, $\partial[0,1]) \rightarrow(V, S)$; the set of the homotopy classes of these mappings is denoted $\tilde{\pi}_{1}(V, S)$; it is isomorphic to $\pi_{1}\left(V, x_{0}\right), x_{0} \in S$.

From now on $\Gamma(f)$ denotes the oriented tree with the system of weights that we have just described, $\alpha_{+}(A), \alpha_{-}(A) \in \tilde{\pi}_{2}(V, S)$ and $\omega\left(A, A^{\prime}\right) \in \tilde{\pi}_{1}(V, S)$.

If $h$ is a homotopy of $f$ to $f^{\prime}$, transversal to $S$ and elementary $\left(h^{-1}(S)\right.$ is an elementary cobordism), and if $f^{\prime-1}(S)$ has less components than $f^{-1}(S)$, then we pass from $\Gamma(f)$ to $\Gamma\left(f^{\prime}\right)$ through one of the following formal operations:

I. collapsing of an edge with a free extremity,

II. folding of two edges, 

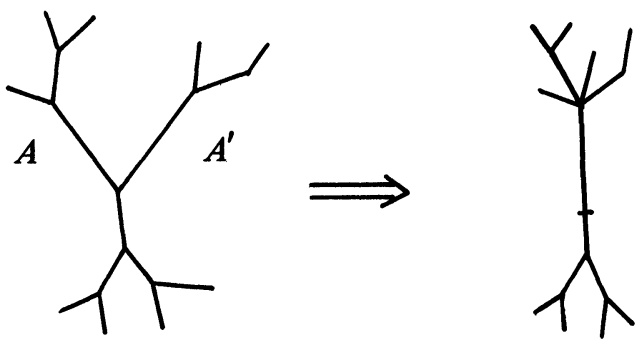

Such a formal operation is said to be admissible if there exists a homotopy of $f$ which realizes it.

We have the following admissability criteria:

Proposition 1. (1) The collapsing of $A$ is admissible iff $\alpha_{\varepsilon}(A)=0$, where $\varepsilon$ is the sign of its free extremity.

(2) The folding of $A_{1}$ and $A_{2}$ is admissible iff $\omega\left(A_{1}, A_{2}\right)=0$.

It is said that $\Gamma(f)$ is reduced if no formal operation on $\Gamma(f)$ is admissible.

Proposition 2. If $f$ and $f^{\prime}$ are two homotopic mappings, transversal to $S$, and if $\Gamma(f)$ and $\Gamma\left(f^{\prime}\right)$ are reduced, then $\Gamma(f)$ and $\Gamma\left(f^{\prime}\right)$ are isomorphic.

Corollary. $f: S^{2} \rightarrow V$, transversal on $S$, is homotopic to $f^{\prime}: S^{2} \rightarrow V-S$ iff $\Gamma(f)$ can be reduced to a point through a succession of admissible formal operations.

Application to prove Theorem I. Let $f: S^{2} \rightarrow V$ be an embedding transversal to $S$. As $f$ is homotopic to $f^{\prime}: S^{2} \rightarrow V-S$, after the corollary we then have either $(\mathrm{H} 1)$ or $(\mathrm{H} 2)$ :

(H1) There exist two curves $C$ and $C^{\prime}$ of $f^{-1}(S)$ such that the folding of the edges $A(C)$ and $A\left(C^{\prime}\right)$ in the graph $\Gamma(f)$ is admissible.

(H2) There exists a curve $C$ of $f^{-1}(S)$ such that the collapsing of $A(C)$ is admissible.

To prove Theorem I by recurrence on card $\pi_{0}\left(f^{-1}(S)\right)$, it is sufficient to prove that, under either hypothesis $(\mathrm{H} 1)$ or $(\mathrm{H} 2)$, there exists an isotopy from $f$ to an embedding $f^{\prime}$, with

$$
\operatorname{card} \pi_{0}\left(f^{\prime-1}(S)\right)<\operatorname{card} \pi_{0}\left(f^{-1}(S)\right)
$$

The case of hypothesis $(\mathrm{H} 2)$ is very classical; it is there that we must suppose that $V$ satisfies the Poincaré conjecture. We use the technique of cuttings and glueings to deal with case (H1). 


\section{BIBLIOGRAPHY}

1. J. Cerf, Sur les difféomorphismes de la sphère de dimension trois $\left(\Gamma_{4}=0\right)$, Lecture Notes in Math., no. 53, Springer-Verlag, Berlin and New York, 1968. MR 37\# 4824.

2. F. Laudenbach, Sur les 2-sphères d'une variété de dimension 3, Centre de Math. de l'Ecole Polytechnique. Ann. of Math. (to appear).

Département de Mathématiques, Université Paris XI, 91-Orsay, France 